Enfermagem Brasil 2018;17(6):585-95

https://doi.org/10.33233/eb.v17i6.1252

\title{
ARTIGO ORIGINAL \\ Validação de orientações para enfermeiros de um serviço de atendimento domiciliar oncológico: estudo metodológico
}

Antonio Jorge Silva Correa Júnior*, Mary Elizabeth de Santana, D.Sc. ${ }^{\star *}$, Lucialba Maria Silva dos Santos, M.Sc. ${ }^{\star \star \star}$, Raisa Silva Martins ${ }^{\star \star \star}$, Érika de Cássia Lima Xavier Barros, M.Sc. ${ }^{\star \star *}$

*Enfermeiro, Mestrando em Enfermagem da Universidade Federal do Pará (UFPA), Belém/PA, **Enfermeira, Professora Titular da Universidade do Estado do Pará (UEPA) e Professor Associado da UFPA, Belém/PA, ${ }^{* * * E n f e r m e i r a ~ U F P A, ~ B e l e ́ m / P A ~}$

Recebido em 18 de outubro de 2017; aceito em 26 de novembro de 2018.

Endereço para correspondência: Antonio Jorge Silva Correa Júnior, Universidade Federal do Pará, Faculdade de Enfermagem, Sala do Mestrado, Rua Augusto Corrêa, Guamá, 66075110 Belém PA, E-mail: juniorjorge_94@hotmail.com; Mary Elizabeth de Santana: betemary@terra.com.br; Lucialba Maria Silva dos Santos: lucialbasilva@hotmail.com; Raisa Silva Martins: raisa-martins@hotmail.com; Érika de Cássia Lima Xavier Barros: erikaxavierteles@hotmail.com

\section{Resumo}

Objetivo: Validar um instrumento que concilie orientações para assistência de enfermeiras e que cite quais os assuntos necessários ao abordar o cuidador de pacientes oncológicos domiciliares. Métodos: Estudo metodológico segmentado em quatro etapas, desenvolvido em um hospital oncológico de referência do município de Belém/PA, entre março e maio de 2015. Resultados: O painel de profissionais foi de seis enfermeiras de unidade de cuidados paliativos que validaram oito orientações gerais. Destas, sete obtiveram consenso apropriado e uma orientação de parecer inadequado (feridas neoplásicas). Educação em saúde, Efeitos adversos e Prevenção de lesões por pressão obtiveram bom desempenho; as demais sofreram aprimoramentos. A orientação que não logrou consenso favorável refletiu a inadequação de produtos descritos em algumas literaturas e a delicada tarefa de universalizar tratamentos, em vista da complexidade das feridas oncológicas. Conclusão: A validação demonstrou que sete orientações estavam adequadas, enquanto uma ainda precisa ser ratificada à prática.

Palavras-chave: enfermagem oncológica, cuidados paliativos, visita domiciliar.

\begin{abstract}
Validation of guidelines for nurses of an oncological domiciliary care services: methodological study

Objective: To validate an instrument that conciliates guidelines for nursing care and which cites the issues required when approaching the caregiver of cancer patients at home. Methods: Methodological study segmented in four stages developed at a reference cancer hospital in the city of Belém/PA, between March and May 2015. Results: The professional panel consisted of six palliative care unit nurses who validated eight general guidelines. Of these, seven obtained appropriate consensus and inadequate guidance (neoplastic wounds). Health education, Adverse effects and Prevention of pressure injuries have performed well; the others suffered improvements. The orientation that did not reach a favorable consensus reflected the inadequacy of products described in some literature and the delicate task of universalizing treatments in view of the complexity of oncological wounds. Conclusion: Validation demonstrated that seven guidelines were adequate, while one still needed to be ratified in practice.
\end{abstract}

Key-words: oncology nursing, palliative care, home visit. 


\section{Resumen \\ Validación de orientaciones para enfermeros de un servicio de atención domiciliar oncológico: estudio metodológico}

Objetivo: Validar un instrumento que concilie orientaciones para asistencia de enfermeras y que cite cuáles son los asuntos necesarios al abordar al cuidador de pacientes oncológicos domiciliares. Métodos: Estudio metodológico segmentado en cuatro etapas y desarrollado en un hospital oncológico de referencia del municipio de Belém/PA, entre marzo y mayo del 2015. Resultados: El panel de profesionales fue de seis enfermeras de unidad de cuidados paliativos que validaron ocho orientaciones generales. De ellos, siete obtuvieron consenso apropiado y una orientación parecer inadecuada (heridas neoplásicas). La educación en salud, los efectos adversos y la prevención de lesiones por presión obtuvieron buen desempeño; las demás sufrieron mejoras. La orientación que no logró consenso favorable reflejó la inadecuación de productos descritos en algunas literaturas y la delicada tarea de universalizar tratamientos, en vista de la complejidad de las heridas oncológicas. Conclusión: La validación demostró que siete orientaciones eran adecuadas, mientras que una aún necesita ser ratificada a la práctica.

Palabras-clave: enfermería oncológica, cuidados paliativos, visita domiciliaria.

Introdução

Estatisticamente, os cuidados paliativos (CP) vêm crescendo e acompanham as mortes por doenças crônicas, impulsionando cuidados multidisciplinares e geralmente contando com planejamento avançado em níveis de ambulatório ou manejo sintomático [1]. A World Health Organization e World Palliative Care Alliance afirmam que os cânceres que demandaram CP causam uma mortalidade de 7.869.752 pessoas ao redor do mundo [2]. No Brasil, durante o biênio 2018-2019, haverá ocorrência de 600 mil casos novos de câncer para cada ano. Os cânceres de próstata em homens e mama em mulheres serão os mais frequentes na população [3].

O CP é a prevenção e o alívio do sofrimento biopsicossocial e espiritual, requerido diante de problemas de saúde graves, crônicos e limitantes de vida, promove assim a dignidade, elevação da qualidade de vida e o ajuste às patologias progressivas utilizando as melhores evidências científicas [4]. O cuidado integral da enfermagem nos CP pode advir de serviços de atendimento domiciliar. A política brasileira Melhor em Casa institui o repasse de informações e o ensino do familiar; e ainda imbui o enfermeiro da avaliação com a escala funcional de Karnofsky (grau de inaptidão ou deficiência funcional), curativos, troca/limpeza de traqueostomia, sondagens, enteroclisma, hipodermóclise, escala visual analógica da dor e execução e ensino de terapias não farmacológicas para dor [5].

A decisão do prognóstico de morte não pode se basear em diagnósticos intuitivos, ao contrário, precisam esmiuçar componentes analíticos e metódicos com recomendações estruturadas, prognósticos baseados em evidências e orientações [6]. Ademais, a política Melhor em Casa afirma que o cuidador familiar é a figura responsável pelo adoecido [5]. Assim, surge a necessidade de um protocolo que embase as ações dos profissionais, com rotinas e ações previstas em um serviço construído por intermédio das evidências científicas [7].

Desta forma, o presente estudo origina-se da necessidade de padronização da assistência de enfermagem e orientações dadas por enfermeiras aos cuidadores durante as visitas de um Serviço de Atendimento Domiciliar (SAD), sediado em um Centro de Alta Complexidade em Oncologia (CACON). Alguns pontos da assistência com vistas no oferecimento de suporte aos cuidadores serão abordados: Anamnese, Educação em saúde, Investigação da rotina, Dor oncológica, Efeitos adversos, Aconselhamento nutricional, Feridas neoplásicas e Prevenção de lesões por pressão. O ensino de cuidados aos familiares é um dos pontos que alicerça os SADs, haja vista que o bom ensino garantirá a longitudinalidade do cuidado prestado na residência.

A falta de informações precisas para cuidadores domiciliares de pacientes oncológicos paliativos reflete-se nos suportes "domínios da informação" e "necessidades de serviços de saúde", que ainda são menos atendidos na visão de cuidadores [8]. Diante disto, o objetivo do estudo é validar um instrumento que concilie orientações para assistência de enfermeiras e que cite quais os assuntos necessários ao abordar o cuidador de pacientes oncológicos domiciliares. 
Trata-se de um estudo metodológico segmentado em quatro etapas. A primeira etapa foi a revisão da literatura nacional e internacional por intermédio de protocolo validado [9], a partir da pergunta norteadora: "Quais as evidências científicas acerca das orientações de enfermagem realizadas ao cuidador familiar do paciente em cuidados paliativos oncológicos domiciliares?". As bases de dados foram a Literatura Latino-Americana e do Caribe em Ciências da Saúde (LILACS), Base de Dados em Enfermagem (BDENF) e Public Medline (PUBMED). Definiram-se conforme os Descritores em Ciências da Saúde (Decs): Cuidados Paliativos, Educação em Saúde, Assistência Domiciliar, Oncologia e Enfermagem, combinados ao termo booleano "and" no ato da pesquisa.

Os critérios de inclusão estabelecidos: artigos disponíveis na íntegra, nos idiomas português, espanhol e inglês; referentes ao período de 2005 a 2014. Excluíram-se: revisões integrativas de literatura, bem como estudos reflexivos e manuais e indicadores institucionais, não houvesse abordagem dos cuidados paliativos domiciliares, nos quais não houvesse menção da abordagem aos CP a pacientes acometidos por neoplasias e ao papel do profissional de Enfermagem. Foram encontradas 849 publicações mediante a aplicação dos critérios de inclusão nas bases, 820 estudos foram excluídos mediante a aplicação dos critérios de exclusão.

O conteúdo das 29 publicações foi explorado e após análise de três revisores, quando se tratam de indícios de orientações prestadas a frequência da primeira análise efetuada foi de $62,06 \%(n=18)$ na amostra $(n=29 / 100 \%)$, não havendo menções as orientações na análise sensível em 37,93\% $(n=11)$. Portanto, foram identificadas em relação ao universo de 18 publicações: Esclarecimento a respeito do alívio da dor com administração de medicamentos, Esclarecimento do prognóstico, Esclarecimento a respeito de medicamentos, sondas, materiais e habilidades para a prestação do cuidado, Esclarecimento a respeito de melhorias quanto à acomodação, adaptação de recursos e horários, Esclarecimento a respeito do manejo de feridas neoplásicas e curativos, Esclarecimento a respeito da troca de decúbito regular para prevenir lesões por pressão e Colaboração na descoberta do significado do que são os cuidados ao paciente oncológico no domić́lio e manutenção da qualidade de vida.

O segundo momento foi de desenvolvimento de orientações para os cuidados aos pacientes oncológicos em cuidados paliativos domiciliares a partir da identificação de assuntos dos 18 artigos, a observância da política Melhor em Casa também foi realizada. Por fim, elaboraram-se 8 orientações gerais (desdobradas em orientações específicas) levadas aos profissionais em um instrumento: Anamnese, Educação em saúde, Investigação da rotina, Dor oncológica, Efeitos adversos, Aconselhamento nutricional, Feridas neoplásicas e Prevenção de lesões por pressão.

A avaliação foi o terceiro momento, ficando a cargo de enfermeiros de unidade de CP por mais de um ano, mediante a assinatura de Termo de Consentimento Livre e Esclarecido. $\mathrm{O}$ painel consultado foi de enfermeiros vinculados à Clínica de Cuidados Paliativos Oncológicos a um hospital de referência do município de Belém/PA que possui $S A D$, e o período da coleta de dados se deu entre os meses de Março e Maio de 2015.

Foi proposta a validação de oito orientações gerais por intermédio da Escala de Likert de cinco pontos: ponto 5 (Muito apropriado), ponto 4 (Parcialmente apropriado), ponto 3 (Nem apropriado nem inapropriado), ponto 2 (Parcialmente inapropriado), ponto 1 (Nunca apropriado). Cada orientação geral desdobrava-se em orientações específicas, no momento da avaliação das orientações gerais, sugestões e adequações foram realizadas pelos participantes.

Após a coleta de dados, no quarto momento, os instrumentos devolvidos foram enumerados com a letra P de "participantes" e sequência de devolução (P1, P2, P3...) e as sugestões tabuladas. Depreendeu-se o cálculo do Índice de Validação de Conteúdo (IVC), por intermédio de tratamento estatístico em frequência simples e percentual com auxílio do programa Microsoft Office Excel. Cuidados recomendados ao atingirem $80 \%(0,8)$ refletirão a adequação e aplicabilidade na prática dos cuidados paliativos domiciliares oncológicos.

Houve incorporações de sugestões e reformulações no campo aberto nas orientações específicas que demandavam adequações a realidade assistencial e condutas pessoais. $O$ estudo foi aprovado pelo Comitê de Ética sob protocolo 23492314.0.0000.0018, atendendo as normas da Resolução № 466/2012. 
O painel de profissionais selecionado compreendeu seis enfermeiras assistenciais de unidade de $\mathrm{CP}$, dentre as quais $33 \%$ possuíam mestrado acadêmico e $67 \%$ pós-graduação lato sensu na modalidade residência multiprofissional atenção em oncologia: cuidados paliativos. Conforme a Tabela I o vislumbre percentual das avaliações.

Tabela I - Avaliação das orientações gerais recomendadas pela Escala de Likert de cinco pontos. Belém/PA, 2016.

\begin{tabular}{lccccc}
\hline Orientações gerais & \multicolumn{4}{c}{ Índices de validação de conteúdo } \\
\cline { 2 - 5 } & $\begin{array}{c}\text { Ponto } \\
\text { Cinco } \\
\mathbf{n}(\%)\end{array}$ & $\begin{array}{c}\text { Ponto } \\
\text { Quatro } \\
\mathbf{n}(\%)\end{array}$ & $\begin{array}{c}\text { Ponto } \\
\text { Três } \\
\mathbf{n}(\%)^{*}\end{array}$ & $\begin{array}{c}\text { Ponto } \\
\text { Dois } \\
\mathbf{n}(\%)^{*}\end{array}$ & $\begin{array}{c}\text { Ponto } \\
\text { Um } \\
\mathbf{n}(\%)^{\star}\end{array}$ \\
\hline Anamnese & $5(83,3)$ & $1(16,6)$ & & \\
Educação em saúde & $6(100,0)$ & & & \\
Investigação da rotina & $5(83,3)$ & $1(16,6)$ & & \\
Dor oncológica & $5(83,3)$ & $1(16,6)$ & & \\
Efeitos adversos & $6(100,0)$ & & & \\
Aconselhamento nutricional & $5(83,3)$ & $1(16,6)$ & $3(50,0)$ & \\
Feridas neoplásicas & $3(50,0)$ & & & \\
Prevenção de lesões por & $6(100,0)$ & & & \\
pressão & & &
\end{tabular}

*frequência absoluta e frequência relativa. Fonte: Instrumento com a Escala de Likert (2016).

Das oito orientações construídas, sete obtiveram IVC adequado acima de 0,8 $(87,5 \%)$, sendo recomendados nove ajustes/acréscimos em três das afirmativas: Investigação da rotina, Dor oncológica e Aconselhamento nutricional; tratando-se das orientações Anamnese, a enfermeira preferiu não registrar comentários. Apenas três permaneceram inalteradas: Educação em saúde, Efeitos adversos e Prevenção de lesões por pressão. Todavia, uma orientação obteve o IVC inadequado à sua validação.

As recomendações com consenso unânime (100\%) foram agrupadas no Quadro 2. O Quadro 3 ordena as orientações gerais que obtiveram pareceres favoráveis, com sugestões e reformulações inclusas nas orientações específicas. Para discernir a natureza das orientações foram empregadas as expressões "orientações para assistência de enfermagem" (OAE) para designar orientações para as enfermeiras, e "orientações para o ensino de cuidadores" (OEC) para designar o repasse de informações sobre os cuidados para os familiares como preconiza a política Melhor em Casa. 
Quadro 1 - Sugestões e críticas referentes as orientações gerais. Belém/PA, 2017.

\begin{tabular}{|c|c|c|c|}
\hline Participante & Orientação geral & Parecer (Likert) & Sugestão \\
\hline $\mathrm{P} 1$ & Feridas neoplásicas & $\begin{array}{l}\text { Nem apropriado nem } \\
\text { inapropriado (3) }\end{array}$ & $\begin{array}{l}\text { Alguns produtos não fazem parte } \\
\text { da instituição e seria inviável o } \\
\text { fornecimento ao familiar, pois o } \\
\text { mau uso pode comprometer a } \\
\text { recuperação. }\end{array}$ \\
\hline \multirow[t]{3}{*}{ P2 } & $\begin{array}{l}\text { Investigação da } \\
\text { rotina }\end{array}$ & $\begin{array}{l}\text { Parcialmente } \\
\text { apropriado (4) }\end{array}$ & $\begin{array}{l}\text { Caso o cuidador seja incapaz de } \\
\text { ler e interpretar informes, como } \\
\text { fazer para que ele possa } \\
\text { administrar a medicação de forma } \\
\text { adequada? Sugestão: desenhar na } \\
\text { caixa do fármaco ou colar } \\
\text { etiquetas com meio Sol para } \\
\text { manhã; Sol inteiro para tarde; e } \\
\text { Lua para noite. }\end{array}$ \\
\hline & Dor oncológica & Muito apropriado (5) & $\begin{array}{l}\text { Acrescentar "Efeitos do } \\
\text { tratamento": } \\
\text { a) Fatores de alívio da dor } \\
\text { b) Fatores de piora da dor }\end{array}$ \\
\hline & Feridas neoplásicas & $\begin{array}{l}\text { Nem apropriado nem } \\
\text { inapropriado (3) }\end{array}$ & $\begin{array}{l}\text { Retirar produtos e acrescentar "se } \\
\text { necessário", a fim de não } \\
\text { universalizar totalmente as } \\
\text { recomendações, haja vista que } \\
\text { cada ferida apresentará sua } \\
\text { particularidade. }\end{array}$ \\
\hline \multirow[t]{2}{*}{ P3 } & $\begin{array}{l}\text { Aconselhamento } \\
\text { nutricional }\end{array}$ & $\begin{array}{l}\text { Parcialmente } \\
\text { apropriado (4) }\end{array}$ & $\begin{array}{l}\text { A avaliação do fonoaudiólogo é } \\
\text { requisitada quando a via de } \\
\text { alimentação é alternativa. }\end{array}$ \\
\hline & Feridas neoplásicas & $\begin{array}{l}\text { Nem apropriado nem } \\
\text { inapropriado ( } 3 \text { ) }\end{array}$ & $\begin{array}{l}\text { Metronidazol macerado é o } \\
\text { indicado para o controle de odores. }\end{array}$ \\
\hline P4 & Dor oncológica & Muito apropriado (5) & $\begin{array}{l}\text { Acrescentar "Sintomas": } \\
\text { a) Desconforto respiratório }\end{array}$ \\
\hline P5 & Feridas neoplásicas & Muito apropriado (5) & $\begin{array}{l}\text { Orientar o cuidador e implementar } \\
\text { condutas com o paciente para } \\
\text { manutenção da integridade da pele } \\
\text { é importante, porém algumas } \\
\text { orientações descritas devem ser } \\
\text { ajustadas. }\end{array}$ \\
\hline P6 & Dor oncológica & $\begin{array}{l}\text { Parcialmente } \\
\text { apropriado (4) }\end{array}$ & $\begin{array}{l}\text { Não se observou sendo realizados } \\
\text { métodos não farmacológicos, } \\
\text { devido ao perfil dos usuários em } \\
\text { CP. }\end{array}$ \\
\hline
\end{tabular}


Quadro 2 - Orientações gerais (IVC = 1) e orientações específicas inalteradas. Belém/PA, 2017.

\begin{tabular}{|l|l|}
\hline $\begin{array}{l}\text { Orientações gerais } \\
\text { IVC=1 }\end{array}$ & Orientações específicas \\
\hline Educação em saúde & $\begin{array}{l}\text { OAE: Intervenção em provável déficit de conhecimento } \\
\text { esclarecendo o prognóstico do tipo de câncer; }\end{array}$ \\
\cline { 2 - 3 } & $\begin{array}{l}\text { OAE: Propiciar instantes para educação em saúde e } \\
\text { treinamento. }\end{array}$ \\
\hline Efeitos adversos & OAE: Esclarecer a incidência de efeitos adversos, comumente; \\
\cline { 2 - 2 } prevenşão & $\begin{array}{l}\text { Orientar sobre a imprescindibilidade de terapias adjuvantes. Ex.: } \\
\text { combater a constipação maligna, náuseas e vômitos, com } \\
\text { antieméticos, laxantes e analgésicos. }\end{array}$ \\
\hline & $\begin{array}{l}\text { OAE: Avaliar risco para úlceras por pressão por meio da escala } \\
\text { de Braden; }\end{array}$ \\
\cline { 2 - 2 } & $\begin{array}{l}\text { OEC: Controlar a incontinência urinária e fecal, orientando } \\
\text { cuidados de higiene após as evacuações e diurese; }\end{array}$ \\
\cline { 2 - 2 } & $\begin{array}{l}\text { OEC: Promover hidratação da pele com produtos tais como } \\
\text { óleos e cremes; }\end{array}$ \\
\cline { 2 - 2 } & OEC: Realização de curativo em caso de úlcera já presente; \\
\cline { 2 - 2 } & $\begin{array}{l}\text { OEC: Orientar cuidador informal com medidas preventivas: } \\
\text { reposicionamento de } 2 \mathrm{~h} / 2 \mathrm{~h} \text { e redistribuição de peso com coxins } \\
\text { e travesseiros em protuberâncias ósseas. } \\
\text { OAE: Dialogar com profissional de medicina em casos de } \\
\text { infecção, para prescrever medicação a fim de reduzir } \\
\text { microrganismos. }\end{array}$ \\
\hline
\end{tabular}

Fonte: Instrumento com a Escala de Likert (2016). 
Quadro 3 - Orientações gerais (1> IVC > 0,8) e orientações específicas com reformulações. Belém/PA, 2017.

\begin{tabular}{|c|c|}
\hline $\begin{array}{l}\text { Orientações } \\
\text { gerais } \\
1>\text { IVC }>0,8\end{array}$ & Orientações específicas \\
\hline \multirow[t]{3}{*}{ Anamnese } & $\begin{array}{l}\text { OAE: Verificar mecanismos adaptativos referentes ao estágio terminal e } \\
\text { luto antecipado; } \\
\text { OAE: Pedir auxílio do profissional de Psicologia. }\end{array}$ \\
\hline & $\begin{array}{l}\text { OAE: Aconselhamento contextualizado sobre as atribuições de cada } \\
\text { membro da equipe e objetivos dos cuidados paliativos oncológicos; }\end{array}$ \\
\hline & $\begin{array}{l}\text { OAE: Perguntar sobre dificuldades no tratamento medicamentoso, } \\
\text { dieta, higiene e quaisquer outras preocupações emergentes. }\end{array}$ \\
\hline \multirow[t]{2}{*}{$\begin{array}{l}\text { Investigação da } \\
\text { rotina }\end{array}$} & $\begin{array}{l}\text { OAE: Estruturar junto ao cuidador horários acessíveis à administração } \\
\text { da medicação conforme o prescrito: } \\
\text { a) Atenção para a identificação correta dos medicamentos, no caso de } \\
\text { cuidadores com letrismo a-funcional, instruí-los por meio de desenhos e } \\
\text { números nas caixas. }\end{array}$ \\
\hline & $\begin{array}{l}\text { OAE: Indagar sobre a necessidade de mudanças na lógica domiciliar, } \\
\text { implementando uma rotina acessível à realidade do cuidador que preze } \\
\text { pelo seu autocuidado haja vista seu desgaste físico e emocional. }\end{array}$ \\
\hline \multirow[t]{5}{*}{ Dor oncológica } & OAE: Avaliação do tipo de dor: nociceptiva, neuropática ou mista; \\
\hline & $\begin{array}{l}\text { OAE: Avaliação da duração da dor: aguda/intermitente ou crônica: } \\
\text { a) Emprego da escala analógica de dor. } \\
\text { b) Fatores de alívio da dor e fatores de piora da dor. }\end{array}$ \\
\hline & $\begin{array}{l}\text { OAE: Métodos farmacológicos } \\
\text { a) Dor leve: Considerar admissibilidade de analgésicos não opioides + } \\
\text { terapia adjuvante; caso refrataria implementar opioides fracos + terapia } \\
\text { adjuvante. } \\
\text { b) Dor moderada: opioides fracos + terapia adjuvante; caso refratário } \\
\text { considerar implementação de opioides fortes. } \\
\text { c) Dor forte: opioides fortes + terapia adjuvante. } \\
\text { * Doses de resgate devem ser prescritas preventivamente, em todos os } \\
\text { quadros de dor. }\end{array}$ \\
\hline & $\begin{array}{l}\text { OAE: Métodos não farmacológicos } \\
\text { Orientar e treinar os cuidadores informais que se sentirem seguros a } \\
\text { implementar terapias não medicamentosas no alívio das dores. Ex.: } \\
\text { métodos de relaxamento, distração, massagens, imaginação dirigida, } \\
\text { toque terapêutico, Terapia Cognitivo-Comportamental, alongamento, } \\
\text { massoterapia, termoterapia e aplicação de frio. }\end{array}$ \\
\hline & $\begin{array}{l}\text { OAE: Desconforto respiratório (dispneia): o manejo sintomático é } \\
\text { realizado com opioides, de forma semelhante ao da dor. }\end{array}$ \\
\hline \multirow[t]{3}{*}{$\begin{array}{l}\text { Aconselhamento } \\
\text { nutricional }\end{array}$} & $\begin{array}{l}\text { OAE: Avaliar a necessidade de terapia nutricional, caso a via oral seja } \\
\text { inviável e caso a caquexia oncológica esteja comprometendo a } \\
\text { qualidade de vida; } \\
\text { OAE: Pedir auxílio ao profissional de nutrição/fonoaudiólogo. }\end{array}$ \\
\hline & $\begin{array}{l}\text { OAE: Dialogar com o cuidador a implementação de sonda nasogástrica } \\
\text { ou nasoenteral; }\end{array}$ \\
\hline & $\begin{array}{l}\text { OAE: Caso a terapia nutricional esteja em andamento: Avaliar a } \\
\text { permeabilidade da sonda, e atentar para queixas do cuidador informal } \\
\text { quanto à êmese e diarreia. }\end{array}$ \\
\hline
\end{tabular}

No Quadro 4 apresentamos as orientações gerais específicas que tem o IVC menor que 0,8 o que denota a importância de reformulações sugeridas pelos participantes, as quais foram inclusas. 
Quadro 4 - Orientação geral com IVC $<0,8$ e orientações específicas com reformulações. Belém/PA, 2017.

\begin{tabular}{|l|l|}
\hline $\begin{array}{l}\text { Orientação geral } \\
\text { IVC }<\mathbf{0 , 8}\end{array}$ & Orientações específicas \\
\hline $\begin{array}{l}\text { Feridas } \\
\text { neoplásicas }\end{array}$ & OAE: Avaliar local da lesão; \\
\cline { 2 - 3 } & $\begin{array}{l}\text { OAE: Avaliar características, exsudação, odor, infecção, sangramento } \\
\text { e bordas, registrando os achados; } \\
\text { a) OAE: Explicar as especificidades das lesões neoplásicas. Os } \\
\text { cuidados visam o controle de sintomas e não a cura; } \\
\text { b) OEC: Utilizar soluções químicas para minimizar odor (metronidazol } \\
\text { macerado, por exemplo); } \\
\text { c) OEC: O sangramento deve ser evitado durante as trocas, } \\
\text { umedecendo-o com soluções antes de sua remoção suavemente; } \\
\text { d) OEC: Conservar úmido o leito da ferida se necessário; } \\
\text { e) OEC: Utilizar, se necessário, pomadas nas bordas e ao redor da } \\
\text { ferida, a fim de proporcionar proteção da pele na região perilesional; } \\
\text { f) OEC: Utilizar, se necessário, produtos para redução do exsudato; } \\
\text { g) OEC: Utilizar se necessário, analgesia sistêmica ou local na troca } \\
\text { de curativo; } \\
\text { h) OEC: Utilizar, se necessário, curativos oclusivos adequados. }\end{array}$ \\
\hline
\end{tabular}

Fonte: Instrumento com a Escala de Likert (2016).

Discussão

Os protocolos não devem restringir a enfermagem em ações premeditadas e inflexíveis [5], pois a educação em saúde informa e implica em uma agenda de visita que elucide pontos específicos, sem deixar de incentivar o diálogo [10]. Por isto compreende-se que a abordagem paliativa ambulatorial do câncer, favorece o trabalho emocional mesclado com a interpretação de sintomas [11].

Houve reformulações sugeridas que envolvem a "Investigação da rotina": a enfermeira não assinalou mudanças estruturais, entretanto atentou-se para cuidadores com letrismo afuncional e a postulação das orientações por intermédio de desenhos. Diante disso, cabe ao profissional auxiliar na descoberta dos sentidos e objetivos dos CP, direcionando-os a um cuidado holístico [12], as táticas que compõem este contexto tangem a elucidação do quadro clínico direcionando mudanças no cotidiano [13].

A "Dor oncológica" pode ser classificada através da escala analógica de dor e tratamento farmacológico com opioides na literatura, tendo em conta o manejo sucedido por anticonvulsivantes e/ou corticoides [14], como terapias adjuvantes enquadradas na orientação geral "Efeitos adversos". Os métodos farmacológicos exigem recomendações e manejo dos efeitos adversos [15], a introdução dos fármacos é uma fase que requer apreensão de especificidades como aceitação e destreza [16]. Assim, uma destas peculiaridades refere-se quando a eficácia das drogas diminui demandando de 1/10 a 1/6 da dose habitual (doses de resgate) [5].

Duas enfermeiras recomendaram acréscimos ao avaliarem "Dor oncológica". Os fatores de melhora e piora foram enquadrados na orientação que antecede o tratamento da dor [17] e desconforto respiratório foi introduzido como nova orientação específica. Afirma-se que o avanço tumoral provoca a dispneia e recomenda-se o uso de opioides para seu controle [5]. A enfermeira P6 não observa terapias não farmacológicas para dores, embora a assistência de enfermagem encontre subsídios para métodos de relaxamento, distração, imaginação dirigida, toque terapêutico, estimulação elétrica transcutânea, terapia cognitivo-comportamental, alongamento, termoterapia e crioterapia $[15,18]$.

Considera-se que a maioria dos opioides culmina em efeitos adversos, a morfina apesar de desencadeá-los permanece como de primeira escolha em razão da fácil condução de sintomas com terapias adjuvantes [19]. Podem ser induzidos por opioides: constipação maligna [16,20,21], náuseas e vômitos [19-21], tontura, prurido, insônia, retenção urinária [21], alopecia, fadiga, neuropatia sensorial e perda de apetite [20]. Os laxantes osmóticos, estimulantes da mucosa e lubrificantes devem estar associados aos opioides, com antieméticos como a metoclopramida, o haloperidol ou ondansetrona [5].

Semelhantemente, outra necessidade é o "Aconselhamento nutricional" que evita o prosseguimento da caquexia oncológica. No consenso o único profissional que assinalou 
Parcialmente apropriado recordou o atendimento do fonoaudiólogo, incumbindo de diagnosticar fisiopatologias da deglutição [22].

Por fim, "Prevenção de lesões por pressão" foi validada com IVC unânime. Percebe-se que 0 cuidado de enfermagem minimiza fatores desencadeantes da lesão por pressão, potencializa o estado nutricional, estimula a hidratação da pele, controla a incontinência urinaria e fecal e protege proeminências ósseas. Avaliar o risco deve ocorrer por intermédio da escala de Braden no domicílio, com reposicionamento e a redistribuição do peso com travesseiros e coxins [23].

Medidas preventivas, orientações e tratamento destas lesões não ocorrem somente no hospital [24], pois a atenção domiciliar regular previne novas lesões e leva à cicatrização em alguns casos [25]. Finalmente, em "Feridas neoplásicas" apenas $50 \%$ assinalaram Nem apropriado nem inapropriado em virtude da preferência por outros produtos, como 0 metronidazol macerado [26] e pela impraticabilidade em universalizações de cuidados.

Sobre a orientação mal avaliada destaca-se a complexidade das feridas neoplásicas, a necessidade de educação permanente e a dificuldade em encontrar subsídios recentes construídos pela enfermagem [27].

Conclusão

A validação demonstrou que as orientações mais bem avaliadas refletem: abordagem sintomática, minimização de dados provenientes do quadro terminal e suporte ao cuidador familiar e educação permanente para os profissionais.

Este estudo está limitado ao baixo número de participantes, devido ao critério de inclusão de profissionais com mais de um ano de assistência, a restrição loco regional e a orientação inadequada (feridas neoplásicas). Outro ponto é que as recomendações validadas aplicam-se somente ao CACON que sediou o estudo, devido à especificidade de algumas sugestões e críticas de profissionais, o que remete o conteúdo validado apenas ao CACON em questão. O estudo identificou problemas que justificaram a modificação da escrita de algumas orientações.

Faz-se necessária uma continuidade da pesquisa, para que as orientações específicas sejam validadas em um âmbito diferente do loco regional e por uma quantidade mais robusta de profissionais.

Agradecimentos

Conselho Nacional de Pesquisa (PIBIC/CNPq).

Referências

1. Etkind SN, Bone AE, Gomes B, Lovell N, Evans CJ, Higginson IJ, Murtagh FEM. How many people will need palliative care in 2040? Past trends, future projections and implications for services. BMC Medicine 2017;15(1):102. https://doi.org/10.1186/s12916-017-0860-2

2. World Health Organization (WHO). Worldwide Palliative Care Alliance (WPCA). Global atlas of palliative care at the end of life. London (UK): WHO; WPCA; 2014. $111 \mathrm{p}$.

3. Instituto Nacional de Câncer José Alencar Gomes da Silva (BR). Coordenação de Prevenção e Vigilância. Estimativa 2018: incidência de câncer no Brasil. Rio de Janeiro (BR): Instituto Nacional de Câncer José Alencar Gomes da Silva; Ministério da Saúde; 2017. $128 \mathrm{p}$.

4. Chair of Palliative Care; WHO Collaborating Centre Public Health Palliative Care Programmes; Worldwide Hospice Palliative Care Alliance; "la Caixa" Banking Foundation. Building Integrated Palliative Care Programs and Services. Gómez-Batiste X, Connor S, eds. 2017. [citado 2018 Mar 31]. Disponível em: http://kehpca.org/wpcontent/uploads/Go\%CC\%81 mez-Batiste-X-Connor-S-Eds.-Building-IntegratedPalliative-Care-Programs-and-Services.-2017-b.pdf.

5. Ministério da Saúde. Secretaria de Atenção à Saúde. Departamento de Atenção Básica. Caderno de Atenção Domiciliar, volume 2. Brasília (DF): Secretaria de Atenção à Saúde; Ministério da Saúde; 2013. 207 p. 
6. Taylor $P$, Dowding $D$, Johnson M. Clinical decision making in the recognition of dying: a qualitative interview study. BMC Palliative Care 2017;16(1):11. https://doi.org/10.1186/s12904-016-0179-3

7. Alves KYA, Salvador PTCO, Tourinho FSV, Santos VEP. Análise do Conceito "Protocolos de Enfermagem" a partir da Visão Evolucionária de Rodgers. Rev Enferm UFPE online 2014;8(1):177-82.

8. Heckel L, Fennell KM, Reynolds J, Osborne RH, Chirgwin J, Botti M et al. Unmet needs and depression among careers of people newly diagnosed with cancer. Eur J Cancer 2015;51:2049-57. https://doi.org/10.1016/i.ejca.2015.06.129.

9. Hopia $\mathrm{H}$, Latvala E, Liimatainen L. Reviewing the methodology of an integrative review. Scand J Caring Sci 2016;30(4):662-9. https://doi.org/10.1111/scs.12327.

10. Bhatnagar T. Palliative home care: A designer's perspective. Indian J Palliat Care 2015;21(2):250-2. https://doi.org/10.4103/0973-1075.156512

11. Back AL, Park ER, Greer JA, Jackson VA, Jacobsen JC, Gallagher ER et al. Clinician roles in early integrated palliative care for patients with advanced cancer. J Palliat Med 2014;17(11):1244-8.

12. Rutz Porto A, Buss Thofehrn M, Dal Pai D, Coelho Amestoy S, de Oliveira Arrieira IC, Rauber Joner $\mathrm{L}$. Visão dos profissionais sobre seu trabalho no programa de internação domiciliar interdisciplinar oncológico: uma realidade brasileira. Av Enferm 2014;32(1):72-9. https://doi.org/10.15446/av.enferm.v32n1.46065

13. Oliveira SG, Garcia RP, Quintana AM, Budó MDLD, Wünsch S, Silveira CL. Dinâmica de organização dos cuidadores familiares do paciente terminal em internação domiciliar. Cienc Cuid Saude 2012;10(4):674-81. http://dx.doi.org/10.4025/cienccuidsaude.v10i4.18310.

14. Lima AD, Maia IO, Costa Junio I, Lima JTO, Lima LC. Pain evaluation in cancer patients admitted to a teaching hospital of the Northeastern region of Brazil. Rev Dor 2013;14(4):267-71.

15. McPherson CJ, Hadjistavropoulos T, Devereaux A, Lobchuk MM. A qualitative investigation of the roles and perspectives of older patients with advanced cancer and their family caregivers in managing pain in the home. BMC Palliat Care 2014;13:39. https://doi.org/10.1186/1472-684x-13-39

16. Prommer EE. Pharmacological management of cancer-related pain. Cancer Control 2015;22(4):412-25. https://doi.org/10.1177/107327481502200407

17. Health Service Executive (HSE). Royal College of Physicians (RCPI). Pharmacological Management of Cancer Pain in Adults: National Clinical Guideline n. 9. National Clinical Effectiveness Committee. Novembro, 2015. Disponível em: http://health.gov.ie/wpcontent/uploads/2015/11/Pharma-Mgmt-Cancer-Pain web.pdf.

18. Pilatto MTS, Rosanelli CP. Medidas não farmacológicas possíveis de serem implementadas por enfermeiros para tratar de pacientes com dor oncológica. [PósGraduação Lato Sensu]. ljuí/RS: Universidade Regional do Noroeste do Estado do Rio Grande do Sul; 2011. 18p.

19. Nunes BC, Garcia JBS, Sakata RK. Morfina como primeiro medicamento para tratamento da dor de câncer. Rev Bras Anestesiol 2014;64(4):236-40. https://doi.org/10.1016/j.bjan.2013.06.018

20. Oliveira PI, Pereira CAC, Belasco AGS, Bettencourt ARC. Comparison of the quality of life among persons with lung cancer, before and after the chemotherapy treatment. Rev Latinoam Enferm 2013;21(3):787-94. https://doi.org/10.1590/s010411692013000300019

21. Cavalcanti IL, Carvalho ACG, Musauer MG, Rodrigues VS, Migon RN, Figueiredo NV et al. Safety and tolerability of controlled-release oxycodone on postoperative pain in patients submitted to the oncologic head and neck surgery. Rev Col Bras Cir 2014;41(6):393-9. https://doi.org/10.1590/0100-69912014006003

22. Conselho Federal de Fonoaudiologia (CFFa). Dispõe sobre a participação do fonoaudiólogo na equipe multidisciplinar de terapia nutricional. Parecer normativo da CFFa, n. 40. [Internet]. 18 fev 2016. [citado 2016 Set 14]. Disponível em: http://www.fonoaudiologia.org.br/cffa/wp-content/uploads/2013/07/parecer-n.-40-2016equipe-multi.pdf.

23. Ministério da Saúde. Agência Nacional de Vigilância Sanitária (ANVISA). Fundação Oswaldo Cruz (Fiocruz). Anexo 02: Protocolo para prevenção de úlcera por pressão. Brasília: ANVISA; Ministério da Saúde; 2013. 21 p. 
24. Moraes JT, Borges EL, Lisboa CR, Cordeiro DCO, Rosa EG, Rocha NA. Conceito e classificação de lesão por pressão: atualização do National Pressure Ulcer Advisory Panel. Rev Enferm Cent-Oeste Min 2016;6(2):2292-306.

https://doi.org/10.19175/recom.v6i2.1423.

25. Sankaran BM, Chakraborty S, Patil VM, Raghavan SN, Thomas S, Sen S. Burden and outcomes of pressure ulcers in cancer patients receiving the Kerala model of home based palliative care in India: Results from a prospective observational study. Indian J Palliat Care 2015;21(2):152-7. https://doi.org/10.4103/0973-1075.156469.

26. Gozzo TO, Tahan FP, Andrade M, Nascimento TG, Prado MAS. Ocorrência e manejo de feridas neoplásticas em mulheres com câncer de mama avançado. Esc Anna Nery 2014;18(2):270-6.

27. Firmino F, Alcântara LFFL. Enfermeiras no atendimento ambulatorial a mulheres com feridas neoplásicas malignas nas mamas. Rev RENE 2014;15(2):298-307.

https://doi.org/10.15253/rev\%20rene.v15i2.3155. 